\title{
ANALYSIS OF ACTUAL PRACTICE OF ACUTE ISCHAEMIC STROKE PHARMACOTHERAPY IN INTENSIVE CARE UNIT: CASE SERIES
}

\author{
Abigail Attard, Esther Muscat
}

University of Malta, Msida

\section{ABSTRACT}

A case study approach was adopted to analyze the pharmacological management of clinical cases of acute ischaemic stroke subsequent to initial treatment with medicinal products that were administered in the acute setting.

The study was conducted in a tertiary care University Hospital, Clinical Centre, Kragujevac, Serbia, during August 2013, where the rationale of medicines prescribing was evaluated in eight cases. The administered medicines were assessed whether they were used in accordance with the recommendations by the National Institute of Clinical Excellence (NICE) guidelines. Appropriate medicines such as aspirin were noted to be used in the management of the most cases and this was in conformation to these guidelines. Deviations from these recommendations included the use of vitamin supplements with antioxidant properties, iron supplements, antibiotics, benzodiazepenes, ranitidine, aminophylline and risperidone. An appropriate indication for administration of these medicines was not established. Additionaly, this study showed that there were instances where an inappropriate route of administration was used.

All observed deviations from the recommended guidelines were not justified, and thus led to the inappropriate use of medicines and suboptimal care. These can be minimized by clear and precise diagnostic data and conformity to the NICE or other available national guidelines.
Key Words: case study, acute ischaemic stroke, NICE guidelines, rationale of medicines prescribing, intensive care unit

\section{INTRODUCTION}

The pharmacotherapy of acute ischaemic stroke is still the matter of controversy. Although there is an increasing trend of treatment with thrombolytics (from $42.6 \%$ of all patients arriving $\leq 2$ hours to a hospital in year 2003 to $77.0 \%$ in year 2013) ${ }^{1}$, with an improvement in clinical outcomes, subsequent therapy with medicines during the stay of patients in an intensive care unit is not clearly in accordance with available guidelines ${ }^{2}$. However, the choice of medicines for secondary prevention of stroke administered during hospitalization significantly affects overall costs of treatment ${ }^{3}$, while the appropriate use of antihypertensives may improve the stroke outcomes ${ }^{4}$. Antioxidant therapy is also frequently prescribed to patients with acute ischaemic stroke, although its efficacy was not confirmed in clinical trials 5 . In these situations, patients with acute ischaemic stroke frequently receive unnecessary medication. The aim of our study was to analyze the actual practice and to understand the rationale for the prescribing of medicines in patients with acute ischaemic stroke during their stay in intensive care unit, using a case study approach.

\section{METHODS}

This case series analysis was conducted in a tertiary care University hospital, Clinical Centre, Kragujevac, Serbia, during August 2013. The 
treatments given to the patients included in this analysis were evaluated in the sense of its compliance with the recommendations by the National Institute of Clinical Excellence (NICE) guidelines ${ }^{6}$. In this analysis we reviewed whether there were deviations in the indication and regimen of a treatment specified in a patient's medical records, in comparison with the recommended guidelines.

\section{RESULTS}

Case 1. A 46-year old male was admitted to the hospital and diagnosed with an acute ischaemic stroke, with left hemiparesis. The main insult was in the right periventricular and parietal zones, shown as little circular hypodense zones. It was also observed upon imaging that he had an old left supratentorial insult. The patient had a history of hypertension and hyperkalemia for which he was given therapy by a nephrologist. He was showing instability in walking during the past five years but he had not consulted a doctor. The patient's data relating to his medical treatment were analyzed on the third day after admission. Ascorbic acid injections $(500 \mathrm{mg} / 5 \mathrm{ml}$ i.m.) were given to the patient every 12 hours. This was not as recommended by the guidelines used for this study, and there was no justification for its administration in an acute ischaemic stroke setting. Ranitidine injections $(50 \mathrm{mg} / 2 \mathrm{ml}$ i.v.) were also given to this patient every 12 hours. It seems that ranitidine was used for gastro protection, in order to prevent stress ulcers, since the patient was being treated with aspirin concomitantly. However, in the guidelines used in this study there is no recommendation of ranitidine use for stress ulcer prevention in stroke patients.

Case 2. A 79-year old male was admitted to hospital diagnosed with an acute ischaemic stroke. He had a history of hypertension, diabetes mellitus and cardiac disease. He was treated with the ascorbic acid $(500 \mathrm{mg} / 5 \mathrm{ml}$ i.m.) every 12 hours, which is not recommended by the guidelines, and its use is not justified in an acute ischaemic stroke setting. Ranitidine $(50 \mathrm{mg} / 2 \mathrm{ml}$ i.v.) was administered on a twice daily $(b d)$ basis, and its use also was not justified, since there was no diagnosis in the patient's medical record for which it would be indicated, and unconditional gastro protection is not recommended by the guidelines. Doses of $350 \mathrm{mg}$ of aminophylline tablets were also used on a $b d$ basis, while there was no diagnosis in the patient's medical record that would justify the use of this medicine with a narrow therapeutic window and high potential for interactions. Moreover, in accordance with the British National Formulary (BNF) ${ }^{7}$, caution should be taken when prescribing Aminophylline to a patient with cardiac disease and hypertension, which were two co-morbidities of this patient. Additionaly, lorazepam was used "when necessary" (prn), and also there was no particular indication for this drug to be used. In this case, no initial treatment for acute ischaemic stroke was taken into consideration, since the patient was seen a day after admission.

Case 3. A 75-year old female was admitted to hospital diagnosed with an acute ischaemic stroke, which resulted in a right hemiparesis. The patient was diabetic and hypertensive and also suffered from dementia. Two ampoules of vitamin C (Ascorbic acid $500 \mathrm{mg} / 5 \mathrm{ml}$ i.m.) were given to this patient twice daily. Doses of 50mg of ranitidine were administered on a twice daily basis $(b d)$, without obvious justification. Gentamicin injections $(120 \mathrm{mg} / 2 \mathrm{ml}$ i.m.) wereadministered on a daily basis. In the patient's file, there was no mentioning of any infection or other possible indication for this antibiotic to be administered, and therefore the use of gentamicin could be considered as unjustified. Additionaly, one milliliter of risperidone (oral solution $1 \mathrm{mg} / \mathrm{ml}$ ), an atypical antipsychotic, was being given once daily in the evening. According to the guidelines, this medicineis not indicated in an acute ischaemic stroke setting. Although this patient suffers from dementia, it was not specified in her medical record whether this was Alzheimer's dementia, for which risperidone may be given. According to the $\mathrm{BNF}^{7}$, Risperidone is used only in aggressive patients with moderate to severe Alzheimer's dementia unresponsive to non-pharmacological approaches or when there is a risk of harm to self or others. It has been reported that Risperidone can cause hypertension, hyperglycaemia and diabetes mellitus and that it should be used with great caution in the elderly. Considering the age of this patient and the fact that she was hypertensive and diabetic, it is hard to find justification for risperidone use in this case. Since this patient was seen 8 days after the admission, her initial treatment was not taken into account.

Case 4. A 79-year old male was admitted to hospital diagnosed with an acute ischaemic stroke and with bronchopneumonia. The patient suffered a right lateral frontoparietal cerebral infarct, and had a medical history of hypertensionin the past. Two ampoules of vitamin $C$ were given to him every 12 hours, than aminophylline tablets $350 \mathrm{mg}$ on a $b d$ basis, and ranitidine in- 
jection $50 \mathrm{mg} / 2 \mathrm{ml}$ i.v. on a twice daily basis $(b d)$. In the patient's medical record there were no diagnosis that would give a reasons for the administration of these medicines. The patient was seen 15 days after admission and therefore his initial treatment was not considered.

Case 5. A 59-year old male was admitted to hospital diagnosed with an acute ischaemic stroke, where the main insult was in the left cerebellum. He had cardiac problems and suffered from ventricular arrhythmias. He also had a past surgical history of intervertebral disc herniation repair, which was performed in 2009. Doses of $50 \mathrm{mg}$ of tetrazepam were given to this patient in the evening on a daily basis. However, the use of tetrazepam was not justified, since the guidelines for acute ischaemic stroke management do not indicate its usage and there was no diagnosis specified in the patient's medical record for which it would be indicated. This patient was seen 11 days after the admission and his initial treatment was notconsidered.

Case 6. An 84-year old female was admitted to hospital diagnosed with an acute ischaemicstroke, due to the frontoparietaloccipital periventricular insult in the supratentorial region. This stroke resulted in a right hemiparesis. She was hypertensive, suffered from Parkinson's disease and had a history of head contusion and hematoma. Two ampoules of vitamin $\mathrm{C}$ and two ampoules of vitamin B6 were given to her every 12 hours. There has been no publication as yet that provides definite evidence that vitamin $\mathrm{C}$ and vitamin B6 usage has any effect on the stroke outcome. This also holds for the administration of a vitamin B complex, which was administered on a $t d s$ basis. Moreover, iron supplements in the form of syrup were given to the patient three times daily. Her treatment was not in accordance with the guidelines for the management of acute ischaemic stroke, and none of the diagnosis was noted in her medical record for which the use of these medicines could have been justified. Furthermore, two grams of ceftriaxone injection were administered intramuscularly once daily, although in the patient's file there was no record of any infection or other possible indication for this antibiotic. This patient was seen 5 days after admission and her initial treatment was not taken into consideration.

Case 7.A 72-year old male was admitted to hospital diagnosed with an acute ischaemic stroke, and a left hemiparesis. The patient suffered from type 2 diabetes mellitus and hypertension.Two ampoules of vitamin $\mathrm{C}$ were given every 12 hours, ranitidine was given intravenously every 12 hours, probable for gastro protection, while a dose of $1 \mathrm{mg}$ of lorazepam was administered on a $t d s$ basis. The use of lorazepam was not justified by the guideline recommendations or by a diagnosis in the patient medical record. Additionaly, risperidone solution was given to the patient on a prn basis, although his age and the fact that he was hypertensive and diabetic make the use of this drug less appropriate in this case. Since the patient data were noted 17 days after admission to hospital, any initial treatment was not considered.

Case 8. A 70-year old male patient was admitted to hospital diagnosed with an acute ischaemic stroke episode, with a left hemiparesis as previous patient. He was hypertensive and suffered from rheumatoid arthritis. Two ampoules of vitamin $\mathrm{C}$ were given every 12 hours, ranitidine was given intravenously every 12 hours, and aminophylline was also administered, without proper justification. Additionaly, ceftriaxone and ciprofloxacin were administered to this patient, but there was no basis on which the use of these antibacterial medicines could be justified. Since the patient data were recorded 10 days after admission, his initial treatment was not taken into account.

\section{DISCUSSION}

In this case series analysis, eight cases of acute ischaemic stroke were evaluated in order to assess the pharmacological management in a hospital setting, after the initial measures in an acute setting had been already taken. It was observed that certain appropriate drugs, such as aspirin, were used in the management of most of these cases. However, deviations from the recommended guidelines were observed and discussed in the previous section. The following are some general observations made on these cases.

In all of studied cases, it was observed that there was the lack of specific diagnosis in the patient's medical record. Therefore, the administration of particular drugs namely vitamin $\mathrm{C}$, vitamin B complex, iron supplements, ranitidine, aminophylline, risperidone, antibiotics and benzodiazepines could not be justified. This renders the treatment of the patient to be suboptimal since it is not patient-centered and cannot be targeted for the specific diagnosis. For instance, there were cases where a patient was reported to have heart disease without any specifications of what his cardiac problems consisted of. The prescription of drugs when appropriate indication 
was not established is one of the most frequent reasons for inappropriate drug use: a recent study of stress ulcer prophylaxis in non-intensive care units has shown that $58 \%$ of all prescriptions were totally inappropriate due to lack of justifiable indication ${ }^{8}$.

Vitamin C was administered to7 patients out of these 8 studied cases, while iron supplements and a vitamin B complex were administered to a particular patient. Such practice is not as recommended by the guidelines used for this study and so their use in an ischaemic stroke setting is not justified. There are no methodologically sound publications as yet that would justify the use of vitamins $\mathrm{B}$ and $\mathrm{C}$ and iron supplements in an acute ischemic stroke setting. In fact, there is no clinical evidence that there is a significant improvement in the clinical outcome. The administration of vitamin supplements could have been justified only if malnutrition or need of nutritional support were reported in the patient's file as this is also stated in the NICE guidelines used for this study. Meta-analyses have shown that supplementation of vitamins with antioxidant properties (including vitamin C) can neither prevent stroke ${ }^{9}$, nor improve clinical outcomes in the patients with acute ischaemic stroke ${ }^{10}$, although there are studies which have found improvements in biochemical parameters of antioxidant capacity ${ }^{11,12}$. Therefore, routine use of vitamin $\mathrm{C}$ or $\mathrm{B}$ vitamins in acute ischemic stroke cannot be recommended ${ }^{10}$.

It was observed that ranitidine was used in seven patients, although its use is not recommended by the guidelines for acute ischemic stroke management. Four of the patients who were used ranitidine were treated concomitantly with aspirin. This could suggest that ranitidine was used for gastroprotection in order to prevent stress ulcers due to aspirin ingestion. However, this is not necessarily to be the case, since there was lack of consistency considering that three of these 7 patients who were treated with ranitidine were not taking aspirin. In addition to this, one patient who was treated with aspirin, was not treated with ranitidine. This indicates that ranitidine was used without justification, especially since it was not evident that it offers gastro protection in acute ischemic stroke patients, and in all of these 7 cases there were no other diagnosis specified in the patient's record that would indicate its use.

In the three studied cases, the prescription of antibiotics (gentanycin, ceftriaxone and ceftriaxone in combination with ciprofloxacin) was not rational, since there were no mentioning of an infection or other possible indication in the patient's medical record for which antibiotic use would be appropriate. Frequent reason for inappropriate prescribing of antibiotics in intensive care units is pyrexia of unknown origin ${ }^{13}$. Moreover, antibacterial agents are not indicated for the management of an acute ischemic stroke. Misuse of antibiotics promotes emergence, selection and spread of resistant pathogens ${ }^{14,15}$.

The use of benzodiazepines in the three studied cases (lorazepam and tetrazapam), was also not recommended by the guidelines for the management of acute ischemic stroke and there were no diagnosis recorded in the patients' files for which the use of these anxiolytics would be indicated.

Aminophylline was also administered without any justification. Additionally, even if there was a diagnosis indicating its possible use, other first-line agents indicated for the same medical condition could be used since the latter would be better tolerated and less toxic and would have less side-effects ${ }^{16}$. Such inappropriate drug use exposes patients to many potential drug side-effects and drug-drug interactions as well as increases hospital costs.

In some of the studied cases, prescribed medicines were administered intravenously, although its oral forms were available and could have been used instead. This also causes an increase in hospital costs since it is a more expensive form of a medicinal product ${ }^{17}$. In addition to this, the oral route is more convenient and less painful for the patient than the intravenous route.

Main limitation of this study is that it was based on patient records only, and responsible physicians were not interviewed for the prescribing rationale. Therefore, prescribing of some of the mentioned drugs could have been justified by some reasons not visible in the records. These circumstances make our conclusions conditional.

\section{CONCLUSION}

An improvement in the management of an acute ischemic stroke patient in a hospital setting could be achieved by clear and precise recording of diagnostic data, in order to choose the treatment which is specifically targeted for the patient. In this way, patient's factors such as comorbidities could be linked with medicine's factors such as contraindications, precautions and adverse effects. Moreover, if the treatment regi- 
men conforms to the recommended guidelines, the inappropriate usage of medicines is significantly reduced, ensuring optimal pharmacological treatment of the patient, optimal care and decrease in hospital costs.

\section{ACKNOWLEDGEMENTS}

The authors are grateful to Prof. Slobodan Jankovic for his help in the design of the study and writing of the paper. Prof. Jankovic was mentor of the authors during their summer visit as part of a research exchange programme at the Clinical Centre Kragujevac, Serbia, in 2013.

\section{ЛИТЕРАТУРА:}

1. Schwamm LH, Ali SF, Reeves MJ, Smith EE, Saver JL, Messe S, Bhatt DL, Grau-Sepulveda MV, Peterson ED, Fonarow GC. Temporal trends in patient characteristics and treatment with intravenous thrombolysis among acute ischaemic stroke patients at get with the guidelines-stroke hospitals. CircCardiovascQual Outcomes 2013; 6(5): 543-9.

2. Kern R, Nagayama M, Toyoda K, Steiner T, Hennerici MG, Shinohara Y. Comparison of the European and Japanese guidelines for the management of ischaemic stroke. Cerebrovasc Dis 2013; 35(5): 402-18.

3. Huang YC, Hu CJ, Lee TH, Yang JT, Weng HH, Lin LC, Lai SL. The Impact Factors on the Cost and Length of Stay among Acute Ischaemic Stroke. J Stroke Cerebrovasc Dis 2012; 22(7): 152-8.

4. Donovan AL, Flexman AM, Gelb AW. Blood pressure management in stroke. Curr Opin Anaesthesiol 2012; 25(5): 516-22.

5. Gilgun-Sherki Y, Rosenbaum Z, Melamed E, Offen D. Antioxidant therapy in acute central nervous system injury: current state. Pharmacol Rev 2002; 54(2): 271-84.

6. National Institute for Health and Care Excellence, United Kingdom. NICE clinical guideline 68. Stroke: diagnosis and initial management of acute stroke and transient ischaemic attack (TIA), 2008, Available at: http://www.nice.org.uk/nicemedia/live/12018/41331/4 1331.pdf, last accessed 7.10.2013.

7. British National Formulary - BNF, March 2013. RCPCH Publications Ltd and the Royal Pharmaceutical Society of Great Britain, 2013.

8. Issa IA, Soubra O, Nakkash H, Soubra L. Variables associated with stress ulcer prophylaxis misuse: a retrospective analysis. Dig Dis Sci 2012; 57(10): 2633-41.

9. Ye Y, Li J, Yuan Z. Effect of antioxidant vitamin supplementation on cardiovascular outcomes: a metaanalysis of randomized controlled trials. PLoS One 2013; 8(2): e56803.

10. Sánchez-Moreno C, Jiménez-Escrig A, Martín A. Stroke: roles of B vitamins, homocysteine and antioxidants. Nutr Res Rev 2009; 22(1): 49-67.

11. Ullegaddi R, Powers HJ, Gariballa SE. Antioxidant supplementation with or without B-group vitamins after acute ischaemic stroke: a randomized controlled trial. JPEN J Parenter Enteral Nutr 2006; 30(2): 108-14.

12. Ullegaddi R, Powers HJ, Gariballa SE. Antioxidant supplementation enhances antioxidant capacity and mitigates oxidative damage following acute ischaemic stroke. Eur J Clin Nutr 2005; 59(12): 1367-73.

13. Ruvinsky S, Mónaco A, Pérez G, Taicz M, Inda L, Kijko I, Constanzo P, Bologna R. Reasons for inappropriate prescribing of antibiotics in a high-complexity pediatric hospital. Rev Panam Salud Publica 2011; 30(6): 580-5.

14. Yezli S, Li H. Antibiotic resistance amongst healthcare-associated pathogens in China. Int $\mathbf{J}$ Antimicrob Agents 2012; 40(5): 389-97.

15. Razine R, Azzouzi A, Barkat A, Khoudri I, Hassouni F, Chefchaouni AC, Abouqal R. Prevalence of hospital-acquired infections in the university medical center of Rabat, Morocco. Int Arch Med 2012; 5(1): 26.

16. Gagne JJ, Maio V, Rabinowitz C. Prevalence and predictors of potential drug-drug interactions in Regione Emilia-Romagna, Italy. J Clin Pharm Ther 2008; 33(2): 141-51.

17. vanWalraven C, Austin P, Naylor CD. Vitamin B12 injections versus oral supplements. How much money could be saved by switching from injections to pills? Can Fam Physician 2001; 47: 79-86. 